\title{
Concentrated growth factor: A effective regenerative tool for soft and hard tissues in periodontics
}

\author{
Shaik Abdul Cader N M A ${ }^{1 *}$, Snophia Suresh ${ }^{2}$, Uma Sudhakar ${ }^{3}$, Navina Ravindran ${ }^{4}$, Roshini Rose A Paul $^{5}$ \\ ${ }^{1}$ Postgraduate Student, ${ }^{2}$ Professor, ${ }^{3}$ Professor and Head, ${ }^{4}$ Senior Lecturer, ${ }^{5}$ Periodontist, Dept. of Periodontology, Thai Moogambigai \\ Dental College and Hospital, Chennai, Tamil Nadu, India
}

*Corresponding Author: Shaik Abdul Cader N M A

Email: nmashaik@gmail.com

\begin{abstract}
Concentrated growth factor is a recent regenerative tool in the field of periodontics and dentistry which consists of numerous amount of growth factors entrapped in a thick fibrin mesh derived from centrifuging the autologous blood in various speed. It has the ability to regenerate both soft and hard tissues. It is an effective, economical, biocompatible and resorbable biomaterial.
\end{abstract}

Keywords: Concentrated growth factor, Growth factors, Autologous platelet concentrates.

\section{Introduction}

Periodontitis results in soft and hard tissue destruction around teeth, resulting the significant destruction of alveolar bone, periodontal ligament and gingiva, and the root cementum. Once the inflammatory aspect of the disease has been controlled, the ultimate goal of periodontal therapy is the regeneration of the destroyed tissues. Periodontal regeneration is defined as the reproduction or reconstitution of a lost or injured part so that the form and function of lost structures is restored. This should be distinguished from the term "new attachment" which describes the formation of new cementum with inserting collagen fibres on a root surface deprived of its periodontal ligament tissue, but does not necessarily describe regeneration of the entire periodontium. Indeed, periodontal regeneration can only be demonstrated histologically whereby the various components of the periodontium can be visualized. ${ }^{1}$

Spontaneous inherent tissue regeneration elicited by the elimination of causal factors. Guided tissue regeneration by the selective guidance of periodontal ligament cell proliferation and Inductive tissue regeneration through recent advances in cellular biology. Growth factors are general term which used to denote a category of naturally occuring proteins that function within the body to market the mitogenesis (proliferation), directed migration and metabolic activity of cells. Thus the three key cellular events in tissue repair are mitogenesis, migration and matrix synthesis and remodelling. ${ }^{2}$ For a growth factor to affect periodontal regeneration, it must be able to stimulate formation of mineralized as well as non mineralized tissues. A combination of growth factors may more effectively stimulate those diverse processes, one factor that promotes mineralizes tissue (bone and cementum) formation, and another factor that stimulates formation of the nonmineralized tissue i.e., periodontal ligament and gingival connective tissue. In some combination, one growth factor can be a competence factor and other one can be a progression factor.

Platelets play a crucial role in hemostasis and wound healing as they are rich source of growth factors including Platelet Derived Growth Factor (PDGF), Insulin like
Growth Factor (IGF), Transforming Growth Factor- $\beta$ (TGF$\beta$ ), which regulate key cellular events in tissue regeneration including cell proliferation, chemotaxis, differentiation and matrix synthesis and that promote tissue repair, angiogenesis, inflammation, and immune response. ${ }^{3}$ The use of Autologous Platelet Concentrates (APCs), which are rich in growth factors, in surgical techniques has emerged as a possible tool to enhance the outcome of the treatment of periodontal defects. APCs include different types of preparations among which the best known are Platelet-Rich Plasma (PRP), Platelet Rich Fibrin (PRF), Plasma Rich in Growth Factors (PRGF) and Concentrated Growth Factor (CGF) Several commercial techniques for obtaining APCs are available, however, their applications have been confusing because each method leads to a different product with different biology and potential uses.

CGF technology has an interesting characteristics, i.e. the easy and speedy one-step preparation of larger, denser and richer growth factors fibrin matrix than the other solid PRPs. There is a presence of fibrin network constituted by thin and thick fibrillar elements with multiple elements trapped among the fibrin network. CGF seems to possess a good regenerative capacity and versatility. ${ }^{4}$

The main feature of the CGF are its an autologous platelet concentrate, without the addition of exogeneous substances in blood and its consistency is an organic matrix rich in fibrin, able to trap platelets, leukocytes and growth factors, elements that play an important role in the regenerative process.

\section{History}

Kingsley first used the term PRP to earmark thrombocyte concentrate during experiments related to blood coagulation. In 1970 PRP was termed as "Fibrin glue". Knighton D R et al. first demonstrated that platelet concentrates successfully promote healing and they termed it as "Platelet-Derived Wound Healing Factors (PDWHF)", 5 In 1997 Whitman D H et al. named their product PRP during preparation but when the end product had a consistency of a fibrin gel and therefore labeled it as "Platelet Gel". ${ }^{6}$ Marx R. E et al. designated all these products as PRP. ${ }^{7}$ Choukroun $\mathrm{J}$ et al. 
developed another form of PC in France which was labeled as PRF, based on the strong fibrin gel polymerization found in this preparation. It was stamped as a "second-generation" platelet concentrate because it was obviously different from other PRPs. ${ }^{8}$ A new concept of CGF was introduced for making CGF from venous blood, rpm in range of 2400-2700 was used to separate cells was given by sacco. The fibrin rich blocks that were obtained were much larger, richer and denser. In 2010 Concept of sticky bone (autologous fibrin glue mixed with bone graft) was introduced by Sohn .W in $2010 .^{9}$

\section{Clasification of platelet concentrates}

According to POSEIDO recommendations

1. I Generation- Platelet Rich Plasma (PRP)

2. II Generation- Platelet Rich Fibrin (PRF)

3. III Generation- Concentrated Growth Factor (CGF)

\section{Concentrated growth factors (CGF)}

It was introduced by Sacco L in 2006 and it is essentially an upgraded version of PRF with a strengthened fibrin matrix and boosted growth factors and cytokines. The easy and speedy one-step preparation of larger, denser and richer growth factors fibrin matrix than the other solid PRPs. There is a presence of fibrin network constituted by thin and thick fibrillar elements with multiple elements trapped among the fibrin network. CGF seems to possess a good regenerative capacity and versatility. ${ }^{10}$

CGF preparation is simple, safe and economic, natural $100 \%$ autologous, thick fibrin matrix contains Leukocytes, Platelets and Growth factors.

\section{Preparation of CGF}

CGF uses variable rpm from 2400-2700 rpm to separate cells in the venous blood, resulting in fibrin rich blocks that are much larger, denser and richer in growth factors than common PRF. This shows better regenerative capacity and higher versatility when using the fibrin rich block. The resulting fibrin clot/block is of a higher quality due to the concentration of fibrinogen, factor XIII and thrombin that is obtained. Factor XIIIa, which is activated by thrombin, cross links the fibrin clot to increase stability, strength and protection against plasmin mediated degradation.

Clinically, this translates to a clot with higher tensile strength (1.5 kg after 1 hour vs $500 \mathrm{gm}$ ), adhesive strength, and decrease in haemostatic time (105 secs vs 360 secs). Besides the tensile fibrin membrane, a red phase of concentrated red blood cells and platelets is also obtained. This is often mixed with either autogenous or other fillers for a more easy to handle and voluminous cavity filling method. Essentially the CGF is an upgraded version of PRF with a strengthened fibrin matrix and boosted growth factors and cytokines.

\section{CGF preparation}

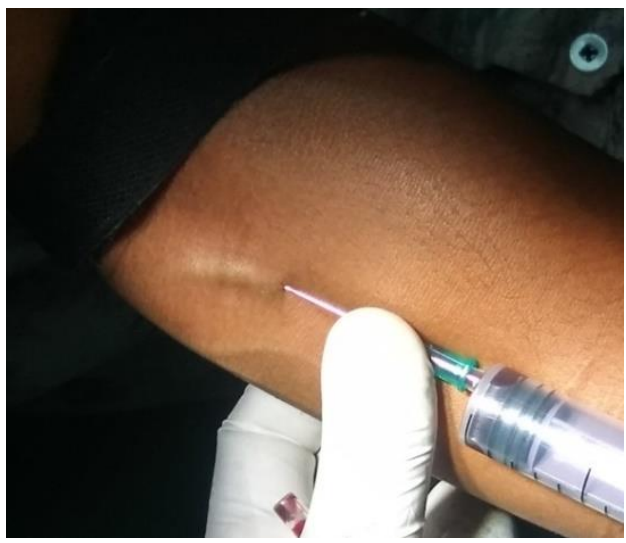

Fig. 1: Venous blood withdrawn using $10 \mathrm{ml}$ syringe

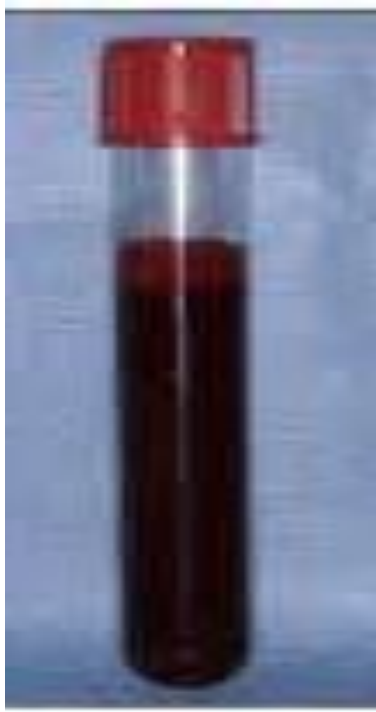

Fig. 2: Venous blood transferred to glass coated plastic tube

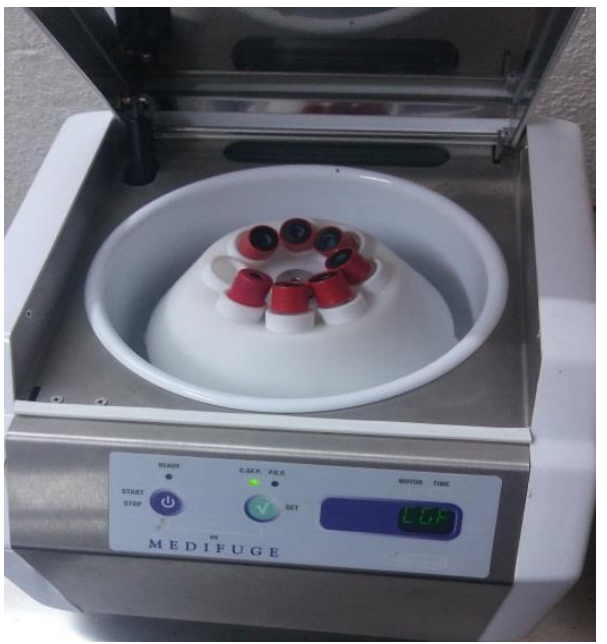

Fig. 3: Collected blood was transferred to Medifuge (silfradent) 


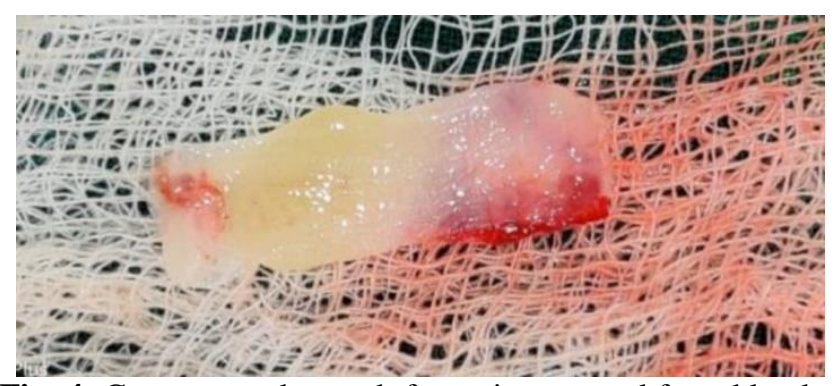

Fig. 4: Concentrated growth factor is procured from blood

\section{The CGF Is characterised by 4 phases: ${ }^{11}$}

\section{Superior phase (Serum)}

Serum is the lightest and most liquid part, it is fibrinogenfree and has only a few cells. It should be kept cool and mixed quickly in order to avoid denaturing the proteins. It is a clear and straw yellow in colour and consists of $92 \% \mathrm{H} 2 \mathrm{O}$, $7 \%$ proteins, mineral, salts, $\mathrm{CO} 2$, albumin, antibodies, glucides, amino acids, lipids, enzymes, hormones and inorganic electrolytes. The serum is used to wash the cavity, to cover and protect all the regenerated portions.

\section{Interim phase (Fibrin Buffy Coat)}

Fibrin block comprises three-dimensional polymer networks with interwoven fibres, all collected in a single phase in the form of a gel.

Inbuilt set up of the equipment guarantee the maximum exploitation of the blood's potential by controlling the following speed, temperature, time, acceleration and controlled speed, and Gravitational acceleration of approximately RCF200. Many corpusculated components are combined, determining numerous therapeutic actions including:

a. Plasma and platelet cytokines: Repair, antiinflammatory and painkilling effect during repair (TNF$\alpha$ ); and

b. Platelets: Transmission of the signals and release of the GFs. The most important are the PDGF-BB, TGFI3 and IGF-1.

Therefore significant volume fibrin gel blocks excellent resistance for: cavity fillers, membrane supports, autologous membranes, and particles to be mixed with another filling material. This translates into a simplified work flow, a high level of regenerative induction and greater versatility of applications for the fibrin block, ranging from the use of the whole block to the particles or membrane.

\section{Liquid phase (Growth factors)}

The Growth Factors and the unipotent Stem Cells are located just below the buffy coat and above the dense clot portion. This phase can be aspirated with a pipette and mixed with autologous bone in order to obtain an extremely high performance activated graft.

\section{Lower phase (Red blood cells)}

This lower phase is dark reddish dense gel. It consists of high concentration of red blood cells and also few white cells, platelets and clotting factors. It is used in pure form or mixed with bone grafts to fill large cavities.

\section{Mechanism}

CGF releases various growth factors such as Plateletderived growth factor (PDGF), Transforming growth factor$\beta 1$ (TGF- $\beta 1$ ) and $\beta 2$ (TGF- $\beta 2$ ), Fibroblast growth factor (FGF), Vascular endothelial growth factor (VEGF), Brain derived growth factor (BDGF) and Insulin-like growth factor (IGF) which stimulate cell proliferation, matrix remodelling and angiogenesis. In vitro study have proved that growth factors like TNF- $\alpha$ and BDGF showed fast kinetic release from the concentrate and reached its maximum accumulation in 1st and 3rd day respectively. Similarly PDGF-AB, TGF- $\beta 1$ and IGF-I had constant kinetic release and reached its maximum in 3rd and 6th day respectively. VEGF and BMP-2 had slow kinetic release and reached its maximum in 8th day. These growth factors predominantly play a role in osteoblast proliferation and differentiation. ${ }^{12}$

\section{Functions}

1. CGF is a fibrin tissue adhesive with haemostatic and tissue sealing properties.

2. It promotes wound healing and accelerates osteogenesis.

3. The CGF improve the wound stability, which is essential for the establishment of a new connective tissue attachment to a root surface.

4. It also provides a scaffold supporting cytokine attachment and cellular migration.

5. Act as a carrier for growth factors. It is an effective surgical haemostatic agent, promotes epithelial, endothelial and epidermal regeneration and decreases dermal scarring.

6. Possess antimicrobial effect due to high concentration of leukocytes.

7. It has anti-angiogenic property on chronic non-healing wounds. ${ }^{13}$

\section{Application of CGF}

CGF is one of the possible methods for reconstruction of soft tissue and bone defects. CGF can be applied alone or mixed with a bone graft. The method is relatively simple, without risk of transmissible and allergic diseases and economically feasible. ${ }^{14}$

\section{General application}

CGF has wide range of healing property in patients undergoing cosmetic surgeries like facelifts, neck lifts, breast augmentation, cardiovascular surgeries, oral and maxillofacial surgeries.

\section{In dentistry}

CGF is used to fill extraction sockets, fill the cavity after cystectomy, sinus lift procedure, ridge augmentation surgeries, recession coverage and also mixed with autologous bone particles or biomaterials to fill the bone 
defects. In implants CGF is used as a membrane support to accelerate bone integration. Potential clinical indications of CGF in oral and maxillofacial surgery are numerous, including, for example, the improvement of soft tissue healing and bone graft protection and remodelling. ${ }^{15}$

\section{Applications in periodontics}

\section{CGF in treatment of gingival recession}

CGF act as a membrane support in recession coverage as it constantly releases growth factors to produce tissue regeneration. Bozkurt Dogan $S(2015)^{16}$ proved that CGF and CAF placed together enhance the healing of soft tissues. The sliding flap technique is significant as this flap has sufficient blood supply for a better healing. CGF membrane provides dual coverage to the exposed root surface. The membrane was exposed in few areas were causes no complications during healing in unexposed areas, with the increase in width of attached gingiva. ${ }^{17}$

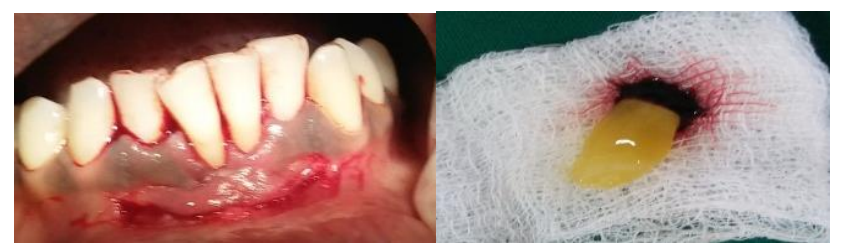

Fig. 5: Recession coverage (Bridge flap) with CGF

\section{Socket preservation or alveolar ridge preservation}

Clinical impact of additional application of CGF with bone graft materials in treatment of bone defects is based on significant radiographic defect bone fill. CGF barrier is effective to regenerate bone formation associated with GBR and GTR procedure. In addition, the mixture of CGF and bone graft could improve healing compared to conventional GBR procedure. ${ }^{18}$

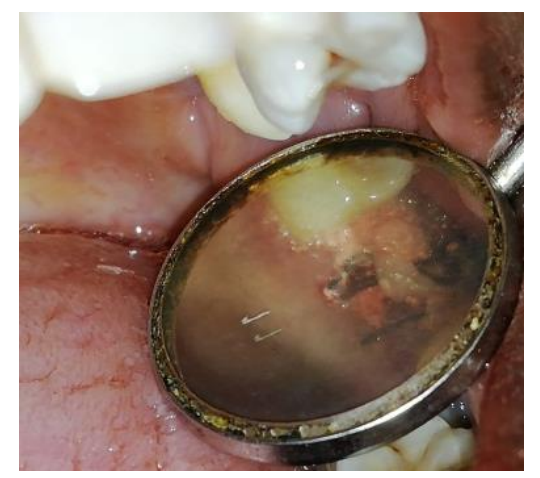

Fig. 6: Socket preservation done with Bone graft (xenograft) and CGF

\section{CGF in treatment of bone defects}

CGF is an excellent bioactive protein which enhances bone healing due to its stimulatory effect on epithelialization and angiogenesis. The advantages of CGF over platelet-rich plasma are lack of biochemical modification, easy method of preparation, application with minimal expense. It also serves as a resorbable interpositional membrane. Avoids the early invigilation of the gingival epithelium is inhibited by the CGF layer results as a barrier to epithelium migration. ${ }^{19}$

\section{a) Effect of CGF on osseointegration}

Osseointegration of dental implants is vital for stability, success and for a longer shelf life. CGF increases implant stability, accelerates osseointegration by increasing the differentiation of osteoblasts and healing of the bone around the implant. ${ }^{20}$

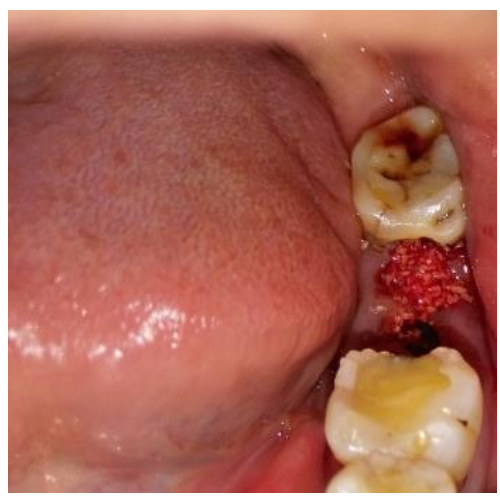

Fig. 7: Bone graft and CGF were kept followed by Implant placement

CGF contains fibrinogen, growth factors, leukocytes, coagulation factors, endothelial growth factor, platelets for angiogenesis and tissue remodeling. It provides a matrix for cell migration. CGF increases FGF- $\beta$ or VEGF release, which are essential for angiogenesis and enhancing neutrophil migration by performing integrin release. Implant cavities are covered with CGF membrane before the implants are placed. ${ }^{21}$ The bone formation is increased in the CGF treated implant site. Pre-implant reconstruction of the deficient alveolar ridge facilitates ideal prosthetic positioning of implants and improves the long-term success of implant-supported restorations. ${ }^{9}$ Addition of CGFs significantly improved clinical effectiveness of Bovine Porous Bone Mineral (BPBM) for intrabony defect treatment. $^{22}$ Concerning specific procedures, CGF may be utilized in combination with graft materials to expedite healing in lateral sinus floor elevation. ${ }^{23}$ Pieri $F$ et al reported on a new technique for maxillary reconstruction using FDBA, PRF, CGF membranes and $0.5 \%$ metronidazole solution. A small quantity of a $0.5 \%$ metronidazole solution $(10 \mathrm{mg})$ was used to provide an efficient protection of the bone graft against unavoidable bacterial contamination. ${ }^{24}$ In addition, all their clinical experiences emphasized that the use of CGF seemed to reduce postoperative pain and edema, and limited even minor infectious phenomena. ${ }^{25}$

\section{Conclusion}

Platelet can play a vital role in periodontal regeneration as they are reservoirs of growth factors and cytokines which are the key factors for regeneration of the bone and maturation of the soft tissue. Platetet- Rich Plasma (PRP) and Platelet Rich Fibrin (PRF) are autologous platelet 
concentrates prepared from patients own blood. Recent researches are being focused on the development of therapeutic alternatives which are easy to prepare, non-toxic or biocompatible to living tissues economically cheap that results in the local release of growth factors accelerating hard and soft tissue healing. On the other hand, concentrated growth factors (CGF), another modified form of PRF, are prepared by repeatedly switching the centrifugation speed and are characterized as a relatively stiffer fibrin clot. The CGF is an autologous platelet concentrate was obtained from blood samples through a simple and standardized separation protocol, by means of a specific centrifuge, without the addition of exogenous substances. The main feature of the CGF resides in its consistency; it is an organic matrix rich in fibrin, able to 'trap' platelets, leukocytes and growth factors; elements that play an important role in the regenerative procedures. Essentially the CGF is an upgraded version of PRF with a strengthened fibrin matrix and boosted growth factors and cytokines. In addition, more studies and clinical trials are needed to investigate potential applications of PRP, PRF and CGF in the field of periodontal regeneration and tissue engineering.

\section{Source of funding}

None.

\section{Conflict of interest}

None.

\section{References}

1. Taba M, Jin Q, Sugai JV, Giannobile WV. Current concepts in periodontal bioengineering. Orthod Craniofac Res 2005;8(4):292-302.

2. Takata T. Oral wound healing concepts in periodontology. Curr Opin Periodontol 1994:119-27.

3. Gassling V, Douglas T, Warnke PH, Açil Y, Wiltfang J, Becker ST. Platelet rich fibrin membranes as scaffolds for periosteal tissue engineering. Clin Oral Implant Res 2010;5439.

4. Bashir B, Jan SM, Behal R, Mir RA, Saima S. Evaluation of efficacy of Concentrated Growth Factor (CGF) biomaterial in the treatment of Intrabony defects -A Randomized Clinical Trial. Int J Curr Adv Res. 2019;8(3)17618-22.

5. Knighton DR, Ciresi K, Fiegel VD, Schumerth S, Butler E, Cerra F. Stimulation of repair in chronic, nonhealing, cutaneous ulcers using platelet-derived wound healing formula. Surg, Gynecol Obstet 1990;170(1):56-60.

6. Whitman DH, Berry RL, Green DM. Platelet gel: an autologous alternative to fibrin glue with applications in oral and maxillofacial surgery. J Oral Maxillofac Surg 1997;55(11):1294-9.

7. Marx RE, Carlson ER, Eichstaedt RM, Schimmele SR, Strauss JE, Georgeff KR. Platelet-rich plasma: Growth factor enhancement for bone grafts. Oral Surg, Oral Med, Oral Pathol, Oral Radiol Endod 1998;85(6):638-46.

8. Choukroun J, Diss A, Simonpieri A, Girard MO, Schoeffler C, Dohan SL, et al. Platelet-rich fibrin (PRF): a second-generation platelet concentrate. Part IV: clinical effects on tissue healing. Oral Surg, Oral Med, Oral Pathol, Oral Radiol Endodontol 2006;101(3):e56-60.

9. Agrawal AA. Evolution, current status and advances in applicationof platelet concentrate in periodontics and implantology. World J Clin Cases 2017;5(5):159-71.
10. Padhayaya VU, Arora A, Goyal A. Bioactive Platelet Aggregates: Prp, Prgf, Prf, Cgf And Sticky Bone. J Dent Med Sci. 2017;16(5);05-11.

11. Mansour P, Kim P. Use of Concentrated Growth Factor (CGF) in implantology. Aust Dent Prac. 2010;162-8.

12. Nityasri, Aromal S, Pradeep KY. Role of Concentrated Growth Factor (CGF) in periodontal regeneration. J Dent Health, Oral Disord Theraphy 2018;9(2):350-2.

13. Del Fabbro M, Panda S, Jayakumar ND, Sankari M, Varghese S, Ramamoorthi S. Autologous platelet concentrates for treatment of periodontal defects (Protocol). Cochrane Database Syst Rev 2014;1-8.

14. Bhanot S, Alex JC. Current applications of platelet gels in facial plastic surgery. Fac Plast Surg 2002;18(1):27-33.

15. Bozkurt Dogan S, O€ngo€zDede F, Ballı U, Atalay EN \& Durmu slar MC. Concentrated growth factor in the treatment of adjacent multiple gingival recessions: a split-mouth randomized clinical trial. J Clin Periodontol 2015;42; 868-75.

16. Ramakrishnan T, Shobana P, Sekhar V, Nirmala JI, Ebenezer, Sivaranjani K. Concentrated growth factor membrane - a novel barrier for accelerated repair of gingival defect along with sliding flap technique. Int J Curr Res Rev 2016;8(21):1-5.

17. Gökmenoğlu, Treatment of Different Types of Bone Defects with Concentrated Growth Factor: Four Case Reports. Int J Oral Dent Health 2016;2(29); 2469-5734.

18. Huang L, Zou R, He J, Ouyang K, Piao Z. Comparing osteogenic effects between concentrated growth factors and the acellular dermal matrix. Braz Oral Res 2018:32:e29.

19. Pirpir. Evaluation of effectiveness of concentrated growth factor on osseointegration. Int J Implant Dent 2017;3:7.

20. Palti A, Hoch T. A concept for the treatment of various dental bone defects. Implant Dent 2002;11(1):73-8.

21. Qiao J, Duan J, Zhang Y, Chu Y, Sun C. The effect of concentrated growth factors in the treatment of periodontal intrabony defects. Future Sci OA. 2016;15:4.

22. Oncu E, Kaymaz E. Assessment of the effectiveness of platelet rich fibrin in the treatment of Schneiderian membrane perforation. Clinical Implant Dent Relat Res.2017;1-6.

23. Pieri F, Lucarelli E, Corinaldesi G, Iezzi G, Piattelli A, Giardino R, et al. Mesenchymal stem cells and platelet-rich plasma enhance bone formation in sinus grafting: A histomorphometric study in mini pigs. J Clin Periodontol 2008;35:539-46.

24. Cheung WS. \& Griffin TJ. A comparative study of root coverage with connective tissue and platelet concentrate grafts: 8-month results. J Periodontol. 2004:75;1678-87.

25. Rodella LF, Favero G, Boninsegna R, Buffoli B, Labanca M, Scari G. Growth factors, CD34 positive cells, and fibrin network analysis in concentrated growth factors fraction. Microsc Res Tech 2011:74;772-7.

How to cite: Cader SANMA, Suresh S, Sudhakar U, Ravindran N, Paul RRA. Concentrated growth factor: A effective regenerative tool for soft and hard tissues in periodontics. IP Int J Periodontol Implantol 2020;5(1):610. 